\title{
Editorial
}

Nephrology

Published online: December 23, 2015

DOI: $10.1159 / 000443243$

\section{Fibroblast Growth Factor 23 and Vascular Calcification: Is It Set in Stone?}

\author{
Gary C.W. Chan Sydney C.W. Tang \\ Division of Nephrology, Department of Medicine, The University of Hong Kong, Queen Mary Hospital, \\ Hong Kong, China
}

The discovery of fibroblast growth factor 23 (FGF23) at the turn of the century had elucidated a new regulatory pathway governing phosphate metabolism and bone biology. A decade later, this circulating phosphaturic hormone is speculated to independently constitute an excess cardiovascular $(\mathrm{CV})$ risk to patients with chronic kidney disease mineral bone disorder (CKD-MBD). In patients with $\mathrm{CKD}$, vascular calcification (VC) is one of the major mechanisms resulting in CV morbidity and mortality. While several small observational studies demonstrated a linkage between higher FGF23 levels and VC $[1,2]$, subcohort analyses of the Chronic Renal Insufficiency Cohort (CRIC), which enrolled approximately 1,500 participants with CKD stages 2-4, refuted the relationship [3]. Kestenbaum et al. [4] subsequently added to the controversy and reported elevated FGF23 to have greater odds of having higher coronary calcium scores in the MESA population. The association of FGF23 with VC is therefore unclear and furthermore, uninvestigated in the African American (AA) population.

In this issue of the American Journal of Nephrology, Freedman et al. [5] performed a cross-sectional analysis on an exclusive cohort consisting of 545 AA patients with type 2 diabetes (T2DM) to investigate the relationship of FGF23 with various parameters of CKD and vascular health [5]. The volumetric bone mineral density, estimated glomerular filtration rate (eGFR), albuminuria and levels of calcified atherosclerotic plaque (CAP) in coronary, carotid and aortic vascular beds were correlated [5]. After adjusting for covariates, analyses revealed an expected negative correlation with eGFR. However, the core finding observed by the investigators was the association of FGF23 with albuminuria and coronary (CAP), but not carotid or aortic CAP. The investigators were able to follow $282(52 \%)$ of the study participants for a mean (SD) of 5.1 (0.9) years. Repeated examinations did not reveal any significant longitudinal correlations.

The patients of this study had fairly preserved renal function (mean eGFR $>90 \mathrm{ml} / \mathrm{min} / 1.73 \mathrm{~m}^{2}$ ) and reverberated previous investigations, demonstrating FGF23 to rise early in the course of CKD. FGF23 also correlated with albuminuria and taken together, the findings suggest this phosphate-regulatory hormone to be a powerful biomarker of incident kidney disease.

There is now a growing body of evidence demonstrating higher FGF23 levels to be associated with increased

Original article editorial based on: Plasma FGF23 and Calcified Atherosclerotic Plaque in African Americans with Type 2 Diabetes Mellitus.

\section{KARGER}

E-Mail karger@karger.com

www.karger.com/ajn
(C) 2016 S. Karger AG, Basel

0250-8095/16/0426-0389\$39.50/0 
CV disease-related death $[6,7]$, questioning provocatively the role played by this hormone. The most noteworthy observation in this study was the correlation of FGF23 with coronary CAP. Although the CRIC study data were non-significant for $\mathrm{VC}$, it should be noted that measures of coronary CAP were not performed in parallel with FGF23 sampling with a median time lag of 376 days. Another striking difference compared to the CRIC study is the utilization of a population with relatively preserved renal function. Freedman et al. were thus able to delineate the relationship of FGF23 with CAP in various vascular beds in isolation, away from the effects of suboptimal phosphorus control inherent to later stages of CKD known to have an impact upon VC [8]. The uniqueness of this study lies in its recruitment of a singular and under-studied cohort of AA participants with T2DM. By virtue of genetic variations, AA race has an altered propensity for CAP and kidney disease [9] and may account for the observed differences in results when compared to CRIC, where only $32 \%$ participants were AA in origin. The results are therefore in concert with that of Kestenbaum et al. [4] and extend the current knowledge on the relationship between FGF23 and coronary CAP in AA patients with T2DM without advanced nephropathy. It is uncertain as to why the coronary vascular bed is affected in isolation and further research is warranted to answer this. However, these results support the negative correlation of FGF23 with peripheral arterial stiffness [10].

Although well performed using an exclusive AA cohort enriched for T2DM with extensive phenotyping, al- lowing for statistical control, the study was limited by a relatively small sample size returning for longitudinal follow-up. This may have hindered any significant capture of the effects of FGF23 on VC. At present, a causal relationship cannot be determined. Whether elevated FGF23 plays a direct role to promote VC or simply occurs as an epiphenomenon in the overall process of CKD-MBD that is associated with CAP remains debatable. The data does, however, provide interesting observations to support future investigations to delineate a potential causal relationship.

Ultimately, this study is a step in the right direction to understanding the part played by FGF23 to the disproportionately high CV morbidity and mortality found in CKD patients. Despite various strategies available to modify conventional CV risk factors, it remains evident that the current armamentarium has had limited impact on this cohort. The implicit corollary is that traditional modifiable $\mathrm{CV}$ risk factors in the general population may not constitute as such in CKD patients. FGF23 may present an alternative pathophysiological avenue of potential target by novel therapeutic strategies to interrupt the attendant CV risk associated with CKD. Future breakthrough will rely on accurate dissection of the role played by FGF23 in vascular health of patients with kidney diseases.

\section{Disclosure Statement}

None.

\section{References}

1 Desjardins L, Liabeuf S, Renard C, et al: FGF23 is independently associated with vascular calcification but not bone mineral density in patients at various CKD stages. Osteoporos Int 2012;23:2017-2025.

2 Schoppet M, Hofbauer LC, Brinskelle-Schmal $\mathrm{N}$, et al: Serum level of the phosphaturic factor FGF23 is associated with abdominal aortic calcification in men: the STRAMBO study. J Clin Endocrinol Metab 2012;97:E575-E583.

3 Scialla JJ, Lau WL, Reilly MP, et al: Fibroblast growth factor 23 is not associated with and does not induce arterial calcification. Kidney Int 2013;83:1159-1168.

4 Kestenbaum B, Sachs MC, Hoofnagle AN, et al: Fibroblast growth factor-23 and cardiovas- cular disease in the general population: the Multi-Ethnic Study of Atherosclerosis. Circ Heart Fail 2014;7:409-417.

5 Freedman BI, Divers J, Russell GB, Palmer ND, Bowden DW, Carr JJ, Wagenknecht LE, Hightower RC, Xu J, Smith SC, Langefeld CD, Hruska KA, Register TC: Plasma FGF23 and calcified atherosclerotic plaque in African Americans with type 2 diabetes mellitus. Am J Nephrol 2015;42:391-401.

6 Scialla JJ, Xie H, Rahman M, et al: Fibroblast growth factor-23 and cardiovascular events in CKD. J Am Soc Nephrol 2014;25:349360.

7 Arnlov J, Carlsson AC, Sundstrom J, et al: Higher fibroblast growth factor-23 increases the risk of all-cause and cardiovascular mortality in the community. Kidney Int 2013;83: 160-166.

8 Adeney KL, Siscovick DS, Ix JH, et al: Association of serum phosphate with vascular and valvular calcification in moderate CKD. J Am Soc Nephrol 2009;20:381-387.

9 Freedman BI, Register TC: Effect of race and genetics on vitamin D metabolism, bone and vascular health. Nat Rev Nephrol 2012;8:459466.

10 Hsu JJ, Katz R, Ix JH, et al: Association of fibroblast growth factor-23 with arterial stiffness in the Multi-Ethnic Study of Atherosclerosis. Nephrol Dial Transplant 2014;29:20992105. 